\title{
Acute ischemic stroke following water immersion seizure: a case report
}

\author{
Kamer Sing Rana ${ }^{1}$, Jyotindra Narayan Goswami², Dinesh Sareen ${ }^{3}$ \\ Departments ${ }^{1}$ Pediatric Neurology and ${ }^{3}$ Neurology, Venkateshwar Hospital, Dwarka; ${ }^{2}$ Department of Pediatrics, Army \\ Hospital (Research \& Referral), New Delhi, India. E-mail : kamersinghrana@yahoo.co.in \\ Received: 14th May 2018, Accepted: 27th June 2018
}

SUMMARY: Sing Rana K, Goswami JN, Sareen N. Acute ischemic stroke following water immersion seizure: a case report. Turk J Pediatr 2019; 61: 453-455.

\begin{abstract}
Water immersion epilepsy or bathing epilepsy is a rare form of reflex epilepsy. We report a 4-year-old child with water immersion epilepsy who manifested with an unusual presentation in the form of acute onset right hemiparesis triggered by sudden splashing of water on his face. The case is an attempt to sensitize clinicians about this entity. It also highlights the role of meticulous history-taking, examination, relevant investigations and management plan in such scenarios.
\end{abstract}

Key words: water immersion seizure, bathing epilepsy, water immersion epilepsy, reflex epilepsy.

Water immersion epilepsy or Bathing epilepsy is a rare form of reflex epilepsy first described by Allen ${ }^{1}$ in 1945. Prevalence is much more in southern India with male predominance. ${ }^{2}$ Bathing, with hot water poured over the head, has been considered a very potent trigger for these seizures. However, many other triggers have been described, including water poured over the body at different temperatures, drops of water touching the face or various body parts, and memories linked to bathing or to other sensory stimuli (e.g., odor of soap, sound of water). ${ }^{2,3}$ Therefore, the term water immersion epilepsy is better than hot water epilepsy or bathing epilepsy. ${ }^{3}$

We report a child, who was having reflex water immersion complex partial seizures since the age of one year. The trigger point was the face and the stimulus was pouring of water irrespective of its temperature. The present episode was a generalized tonic clonic seizure followed by ischemic arterial cortical stroke. Possible pathogenesis of the stroke was probably severe reflex spasm of the cortical end arteries due to pouring of water over the face.

\section{Case Report}

A 4 years old right handed, developmentally normal boy was brought to emergency room with complaints of acute onset weakness of right sided limbs noted about an hour prior. The child was attending playschool where during swimming period, he was asked by the class attendant to enter the swimming pool along with his class-mates. As the child was scared of swimming, he started crying and attempted to run away. The class teacher splashed water on the child's face with a hose. Within seconds, the child sat down, bending his head forward and hiding his face in his hands. Subsequently, he fell on the ground with repetitive jerky movements of limbs, noisy breathing and frothing from mouth. He persistently stared to his right and didn't respond to verbal command and touch. This episode lasted about 1 to 2 minutes. By 30 minutes he was brought to the hospital. On arrival, his vital parameters were normal. He was sleeping but arousable. There was no external injury, neck-rigidity, fever or abnormal odor. Pupils were normally reactive to light. On stimulation, spontaneous movements were absent on right upper and lower limb. Emergency treatment offered 
included iv fluids (dextrose saline), oxygen and levetiracetam $(20 \mathrm{mg} / \mathrm{kg}$ over $30 \mathrm{~min})$. Child's random blood sugar was $110 \mathrm{mg} / \mathrm{dl}$. Detailed examination subsequently revealed motor aphasia, right upper motor neuron VII ${ }^{\text {th }}$ nerve palsy, right hemiplegia (power grade $0 / 5$ in upper limb, 1/5 in lower limb). Other systems were normal. Laboratory parameters suggested leukocytosis. Magnetic resonance imaging (MRI) brain suggested acute ischemic infarct left middle cerebral artery territory (Fig. 1).

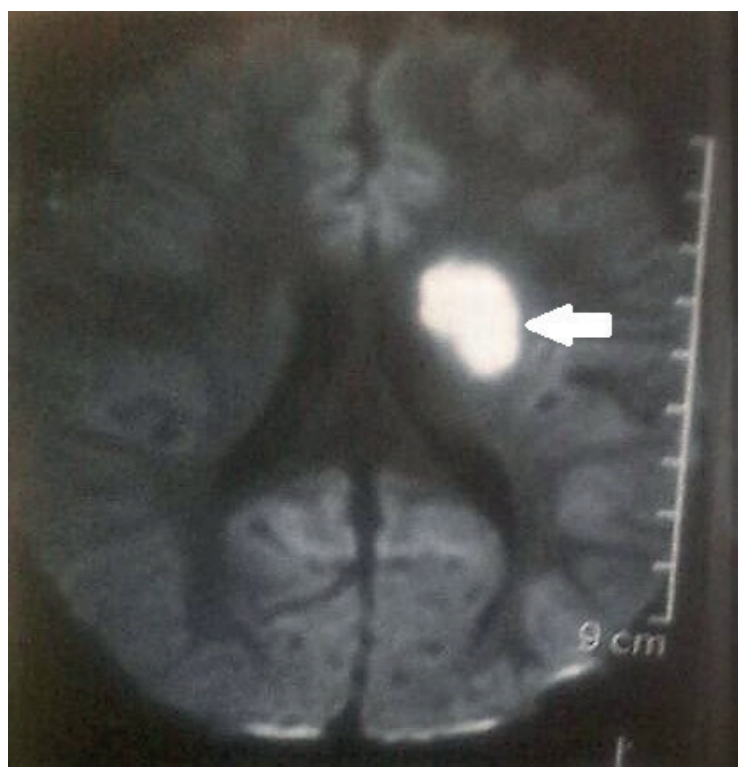

Fig. 1. MRI Brain of the child showing area of intense diffusion restriction (arrow) on Diffusion Weighted Imaging (DWI) in deep left periventricular white matter.

Parents gave history that child used to behave abnormally whenever cool/warm water was poured on his face during bathing since one year of age. He used to remain awake but talked irrelevantly and had repetitive chewing movements, blank stare with no fall. Each episode lasted for about a minute. Hence parents refrained from pouring water on child's face as a precautionary measure and did sponging instead. Similar episodes did not occur on pouring water on head or other body parts. Parents did not let the child to go out in the rain .However they had not shared this history with school authorities previously. Child's developmental history was normal and three generation family history unremarkable. He did not have history of febrile seizures.
Further workup included Magnetic Resonance Angiography (MRA) of neck vessels and brain, electroencephalogram (EEG), echocardiography, hemoglobin electrophoresis which were within normal limits.

The child was managed with acetyl salicylic acid (5mg/kg/day), syrup levetiracetam (20mg/ $\mathrm{kg} /$ day divided 12 hourly) and physiotherapy. He started improving and was discharged after five days on oral levetiracetam, acetyl salicylic acid and physiotherapy. At six-month followup, his speech was normal, he could walk without support. Power in right lower limb had improved to $4 / 5$ and right upper limb to $3 / 5$. His procoagulant work up was negative. Parents were advised to continue levetiracetam, acetyl salicylic acid and physiotherapy. Child was not brought for further follow-up.

\section{Discussion}

Cases of water immersion epilepsy have been reported from different parts of the world. Hot Water Epilepsy (HWE) is a variant of water immersion epilepsy where seizures are provoked by pouring of warm water $\left(45^{\circ}\right.$ to $50^{\circ} \mathrm{C}$ ) over the head though there are reports of other parts of the body including genital organs acting as trigger zones. ${ }^{2}$ The term 'bathing epilepsy' has been suggested by Kowac, et al. ${ }^{3}$ to replace the term 'Hot Water Epilepsy' in view of patients who manifest seizures with warm rather than hot water. This entity has been more frequently observed in South Indian states due to the ritual of bathing with hot water $\left(>45^{\circ} \mathrm{C}\right)$ and pouring of water initially over the head. The incidence of hot water epilepsy is about $6.9 \%$ amongst all epilepsies in South India. ${ }^{4}$ Incidence is more in males (male to female ratio 2.1:1). These seizures may be tonic, clonic, generalized tonic clonic, simple or complex partial precipitated during bathing with warm and rarely cool water in a tub or while taking a shower. ${ }^{5}$ Triggers for this epilepsy includes duration of bathing, amount of water, sound of water, raindrops touching face. ${ }^{4}$ Infants and toddlers may become unresponsive or lethargic with blank stare and bluishness of lips followed by excessive sleepiness or irritability while older children generally have aura in the form of fearfulness, giddiness, confusion, 
hallucinations or automatism. Duration is usually 1 to 2 minutes. Water immersion epilepsy is common in children with history of febrile seizures and family history of epilepsy. Compulsive behaviour of pouring water to self-induce seizure has been reported in certain patients who derive pleasure from this activity. ${ }^{4}$

The exact pathogenesis of water immersion epilepsy is still not known. Mechanisms hypothecated to be responsible for this epilepsy include malregulation of body's thermostat combined with genetic predisposition and a kindling phenomenon. ${ }^{4}$ Loci at chromosomes 10 and 4 have been incriminated in HWE patients from South India. ${ }^{6}$ About 7 to $10 \%$ of the hot water epilepsy are familial and they are transmitted in autosomal dominant fashion. Author has seen four cases of familial water immersion epilepsy, three from south and one from north (unpublished).

Neuroimaging is usually normal in majority of the cases. Inter-ictal EEG is usually noncontributory while Ictal EEG usually indicates an ictal focus at medial temporal or frontal lobe. ${ }^{4}$ Functional neuroimaging(inter--ictal and ictal single-photon emission computed tomography (SPECT) scans) show foci of hyper metabolism in medial temporal lobe of the individuals with hot water epilepsy. ${ }^{4}$

The avoidance of the specific trigger is the best way to treat water immersion epilepsy. ${ }^{7}$ Avoiding initial contact over the trigger zone such as head and initial pouring of water on the other parts of the body and subsequent headsponging can be practiced. The index child had seizures secondary to exposure of the face. Routine anti-epileptics are not prescribed for water immersion epilepsy. However, in case of additional seizures, drugs like carbamazepine , oxcarbazepine, levetiracetam or lacosamide need to be prescribed. Intermittent prophylaxis with clobazam 2-3 hours prior to bathing is advocated in cold places where frequency of bathing is less. ${ }^{7}$
Stroke following water immersion seizure has not been reported. Hemiparesis in the index child could be hypothesized to be secondary to unusual long cerebral arterial spasm following forceful cold water pouring over the face of a child, who was a known to have water immersion epilepsy. In such situations, other probable causes of ischemic stroke should be ruled out before attributing the stroke to reflex water immersion epilepsy as was done in the index child.

To conclude, we report this unusual manifestation of water immersion epilepsy in order to sensitize readers about this entity. We also wish to highlight the role of meticulous history-taking, examination, relevant investigations and management plan in such scenarios.

An informed consent form was signed by the parents of the patient to approve the use of patient information or material for scientific purposes.

\section{REFERENCES}

1. Allen IM. Observations on cases of reflex epilepsy. $\mathrm{N}$ Z Med J 1945; 44: 135-142.

2. Syed R. Hot water epilepsy: A rare form of reflex epilepsy. J Neurosci Rural Pract 2010; 1: 99-101.

3. Kowacs PA, Marchioro IJM, da Silva EB Jr, Rocha SFB da, Simão CA, Meneses MS. "Hot-water epilepsy", "warm-water epilepsy", or bathing epilepsy? Report of three cases and considerations regarding an old theme. Arq Neuropsiquiatr 2005; 63: 399-401.

4. Bebek N, Baykan B, Gürses C, Emir Ö, Gökyiğit A. Selfinduction behavior in patients with photosensitive and hot water epilepsy: A comparative study from a tertiary epilepsy center in Turkey. Epilepsy Behav 2006; 9: 317-326.

5. Auvin S, Lamblin MD, Pandit F, Bastos M, Derambure P, Vallée L. Hot water epilepsy occurring at temperature below the core temperature. Brain Dev 2006; 28: 265-268.

6. Ratnapriya R, Satishchandra P, Kumar SD, Gadre G, Reddy R, Anand A. A locus for autosomal dominant reflex epilepsy precipitated by hot water maps at chromosome 10q21.3-q22.3. Hum Genet 2009; 125: 541-549.

7. Satishchandra P. Hot-water epilepsy. Epilepsia 2003; 44(Suppl 1): 29-32. 